\section{Metal-responsive transcription factor (MTF-1) handles both extremes, copper load and copper starvation, by activating different genes}

\author{
Anand Selvaraj, ${ }^{1}$ Kuppusamy Balamurugan, ${ }^{1,4}$ \\ Hasmik Yepiskoposyan, ${ }^{1,4}$ Hao Zhou, ${ }^{2,4}$ \\ Dieter Egli, ${ }^{1}$ Oleg Georgiev, ${ }^{1}$ Dennis J. Thiele, ${ }^{3}$ \\ and Walter Schaffner ${ }^{1,5}$ \\ ${ }^{1}$ Institute of Molecular Biology, University of Zurich, \\ CH-8057, Zurich, Switzerland; ${ }^{2}$ Howard Hughes Medical \\ Institute, Children's Hospital Boston, Harvard Medical \\ School, Boston, Massachusetts 02115, USA; ${ }^{3}$ Department of \\ Pharmacology and Cancer Biology, Sarah W. Stedman \\ Nutrition and Metabolism Center, Duke University Medical \\ Center, Durham, North Carolina 27710, USA
}

From insects to mammals, metallothionein genes are induced in response to heavy metal load by the transcription factor MTF-1, which binds to short DNA sequence motifs, termed metal response elements (MREs). Here we describe a novel and seemingly paradoxical role for MTF-1 in Drosophila in that it also mediates transcriptional activation of $C \operatorname{tr} 1 B$, a copper importer, upon copper depletion. Activation depends on the same type of MRE motifs in the upstream region of the Ctr1B gene as are normally required for metal induction. Thus, a single transcription factor, MTF-1, plays a direct role in both copper detoxification and acquisition by inducing the expression of metallothioneins and of a copper importer, respectively.

Supplemental material is available at http://www.genesdev.org.

Received January 31, 2005; revised version accepted March 4, 2005.

Copper is an essential trace element that serves as a catalytic cofactor for several enzymes that are mainly involved in respiration, iron transport, and oxidative stress protection (Puig and Thiele 2002). However, an excess of copper ions can catalyze cytotoxic reactions; thus, every organism must be able to tightly regulate copper levels (Halliwell and Gutteridge 1985). Copper imbalance in humans is the cause of serious diseases, such as Menkes syndrome and Wilson disease, and has also been implicated in Alzheimer's disease and prion-type diseases (Harrison and Dameron 1999; Waggoner et al. 1999; Mercer 2001). Copper homeostasis can be regulated at the level of copper uptake, distribution, chelation, and ex-

[Keywords: Transcription factor MTF-1; metal response elements; metallothioneins; Ctr1B; copper load; copper depletion]

${ }^{4}$ These authors contributed equally to this work.

${ }^{5}$ Corresponding author.

E-MAIL walter.schaffner@molbio.unizh.ch; FAX 41-1-6356811.

Article and publication are at http://www.genesdev.org/cgi/doi/10.1101/ gad.1301805 port (Askwith and Kaplan 1998; Culotta et al. 1999). The cellular proteins that are involved in copper homeostasis, such as importers, exporters, and scavengers, are regulated by different mechanisms including transcriptional activation or repression, changes in protein stability, and the modulation of protein trafficking (Petris et al. 2003; Bertinato and L'Abbe 2004; Lane et al. 2004).

From insects to mammals, heavy metal detoxification is controlled to a large extent by the zinc finger transcription factor MTF-1 (metal response element-binding transcription factor-1, also referred to as metal-responsive transcription factor, or just metal transcription factor) (Westin and Schaffner 1988; Radtke et al. 1993; Langmade et al. 2000; Giedroc et al. 2001; Lichtlen and Schaffner 2001; Zhang et al. 2001). Metal response elements (MREs) of consensus TGCRCNC (where R stands for A or $\mathrm{G}$ and $\mathrm{N}$ for any of the four bases) are cis-regulatory DNA sequences that specifically bind MTF-1 and are essential and sufficient for transcriptional induction upon heavy metal load (Stuart et al. 1985; Westin and Schaffner 1988). Major target genes of MTF-1 are the genes encoding metallothioneins-short, cysteine-rich proteins that have the ability to bind and thereby sequester heavy metals (Kägi and Kojima 1987; Palmiter 1998). In the mouse, $M T F-1$ is an essential gene, the knockout of which results in embryonic lethality due to liver degeneration (Günes et al. 1998). The strong up-regulation of the transcription of metallothionein genes upon heavy metal load was abrogated in MTF-1 knockout cells (Heuchel et al. 1994; Günes et al. 1998). A conditional knockout of MTF-1 in the mouse liver produced no phenotype in normal laboratory conditions, but mice were more susceptible to cadmium toxicity (Wang et al. 2004). As in the case of mammals, in Drosophila a major function of the MTF-1 (dMTF-1) is in the activation of metallothionein genes in response to heavy metal load (Zhang et al. 2001; Egli et al. 2003). There are four metallothionein genes in Drosophila, each harboring multiple MREs in their enhancer/promoter region. However, unlike the situation in the mouse, knockout of $d M T F-1$ is not lethal in Drosophila. The mutant flies $\left(d M T F-1^{140-1 R}\right)$ survive well under laboratory conditions but are extremely sensitive to elevated levels of heavy metals including zinc, copper, and cadmium. Consistent with the phenotype, exposure of $d M T F-1$ mutants to heavy metal load failed to induce metallothionein genes (Egli et al. 2003; Balamurugan et al. 2004).

In light of the established role of MTF-1 under conditions of heavy metal load, it came as a surprise that in Drosophila, MTF-1 mutants also died at larval stages when challenged with nutritional copper scarcity (Egli et al. 2003). This seeming paradox prompted us to investigate the role of MTF-1 during copper starvation. We conducted microarray analysis and identified the copper importer $C t r 1 B$ as a potential target gene of dMTF-1. There are three Ctr-type copper transporters in Drosophila, namely, Ctr1A, Ctr1B, and Ctr1C (Zhou et al. 2003). Ctr1B function is important during larval stages, where efficient copper uptake is essential for rapid growth. $C \operatorname{tr} 1 B$ knockout flies $\left(C \operatorname{tr} 1 B^{3-4}\right)$ survive well in normal laboratory conditions but are extremely sensitive to nutritional copper scarcity and, to a lesser degree, also to copper load. The sensitivity of the mutants to copper depletion is consistent with the copper uptake function 
of Ctr1B. It was speculated that the sensitivity of the mutants to copper load was due to an inability to mobilize copper to a potential copper-dependent protein or a storage tissue (Zhou et al. 2003).

Here we demonstrate that the lethal phenotype of dMTF-1 mutants under copper insufficiency conditions is due to the failure of regulating the copper importer Ctr1B. Interestingly, the upstream regulatory region of the $C \operatorname{tr} 1 B$ gene contains MREs that conform to the consensus found in metallothionein genes. By genetic and biochemical analyses we show that these MREs are, however, not used for induction upon copper load, but are essential for the activation of $C \operatorname{tr} 1 B$ by dMTF- 1 under conditions of copper scarcity. Thus, we reveal a novel mechanism whereby a single transcription factor, dMTF1 , plays a central role in both copper detoxification and acquisition, by directly activating transcription of metallothioneins and a copper importer, respectively.

\section{Results and Discussion}

It was known from the $d M T F-1$ knockout study that the Drosophila larvae were not only sensitive to excess copper, zinc, and cadmium but also highly sensitive to copper depletion, as tested by supplementing the food with the specific copper chelator bathocuproinedisulfonate (BCS) (Egli et al. 2003). To understand this phenotype, we assessed the transcriptome response in a deletion mutant of the heavy metal regulator dMTF-1 (dMTF-1 $\left.{ }^{140-1 R}\right)$. A comparison of microarray data from the $d M T F-1$ mutant and wild type (WT) larvae revealed that transcripts of one of the copper importers, $C \operatorname{tr} 1 B$, were reduced in the $d M T F-1$ mutant, whereas expression of the related genes $C \operatorname{tr} 1 A$ and $C \operatorname{tr} 1 C$ was not affected (data not shown). A microarray analysis of genes up-regulated in low copper conditions in wildtype Drosophila, on the one hand, and genes with decreased expression in the $d M T F-1$ deletion mutant in normal food, on the other, revealed $C \operatorname{tr} 1 B$ as the only overlapping gene (Fig. 1A). These findings were confirmed by RNA blotting, which showed in wild-type Drosophila, an opposite regulation of the $C \operatorname{tr} 1 B$ gene as compared with a well-characterized target gene of MTF-1, metallothionein A (MtnA). While the latter was strongly induced by excess copper in the food, $C \operatorname{tr} 1 B$ was at the same time down-regulated, but induced by copper chelator treatment. In the $d M T F-1$ mutant, the $M t n A$ transcripts were not detectable at any condition, while $C \operatorname{tr} 1 B$ transcripts were reduced in normal food and could no longer be up-regulated in response to copper chelator treatment (Fig. 1B).

The loss of regulation of $C \operatorname{tr} 1 B$ in the $d M T F-1$ mutant prompted us to test whether Ctr1B was responsible for the unexpected sensitivity to copper deprivation of the $d M T F-1$ mutant Drosophila. For this, we attempted to shortcut the regulation by constitutively overexpressing Ctr1B. Several transgenic fly lines were generated with a $C \operatorname{tr} 1 B$ ORF driven by $\mathrm{UAS}_{\mathrm{GAL}}$. The Ctr1B transgenic flies survived well but invariably died if crossed with flies constitutively expressing the Gal4 transcription factor via the actin5c promoter. To test whether this lethality was due to uncontrolled copper import or another effect, we raised the larvae in food with increasing amounts of copper chelator. The results were clear, in that the flies survived only in the presence of high chelator concentrations, while wild-type flies survived under all conditions (Fig. 1C). These observations suggest that the larvae died from copper toxicosis, even in normal food, due to the strong, ectopic expression of Ctr1B. We used the same system to test whether this constitutive expression of Ctr1B could rescue the lethal phenotype of the dMTF-1 mutant under low copper conditions. dMTF-1 mutant Drosophila are developmentally arrested and die at second or third instar larval stages when the concentration of BCS reaches $50 \mu \mathrm{M}$ in the food. Strikingly, constitutive Ctr1B expression rescued the developmental arrest and larval lethality of the $d M T F-1$ mutants under copper depletion, and several viable $d M T F-1$ mutant flies were obtained from food containing 50 or even 100 $\mu M$ BCS (Fig. 1D; Supplementary Table 1). The rescued $d M T F-1$ mutant flies were normal and fertile (Supplementary Fig. 1; data not shown). The constitutive Ctr1B expression and lack of tissue specificity probably prevented a complete rescue of $d M T F-1$ mutants in all concentrations of BCS tested.

While these results demonstrated that $C \operatorname{tr} 1 B$ is an essential downstream target gene of dMTF-1 under copper
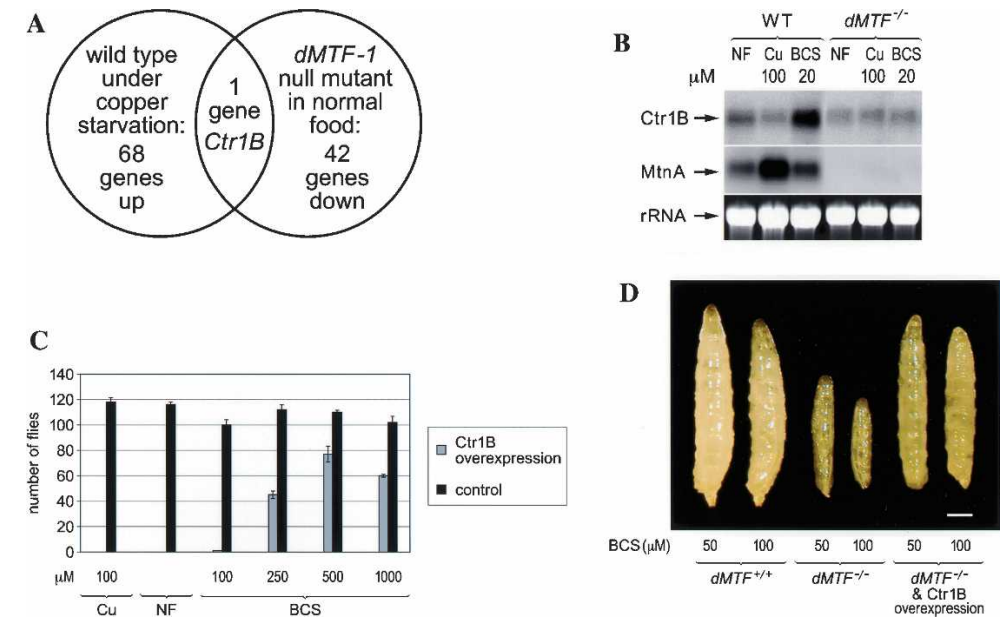

Figure 1. Drosophila copper importer $C \operatorname{tr} 1 B$ is a target gene of MTF-1. (A) Summary diagram of microarray experiments to identify dMTF-1 target genes in copper depletion (the complete microarray data will be presented elsewhere). Sixtynine genes were up-regulated more than twofold when wild-type larvae fed with BCS (copper chelator) containing food were compared with larvae fed with normal food ( $p$-value $<0.05)$. In Drosophila larvae lacking $d M T F-1,43$ genes were downregulated in normal food, when compared with wild-type controls $(p$ value $<0.05)$. Ctr1B was the only overlapping gene in these two experimental conditions. (B) Regulation of Ctr1B upon copper starvation is lost in the absence of $d M T F-1$. RNA blotting analysis of total RNA obtained from third instar larvae at different conditions. (WT) Wild type; (NF) normal food; (rRNA) reference. $(C)$ Lethality of Ctr1B overexpression in normal food is rescued by BCS. Ctr1B overexpression, y $\mathrm{W}$; actin-Gal4/UAS-Ctr1B; control, y w; +/UAS-Ctr1B. y w; UASCtr1B/UAS-Ctr1B (homozygous) flies were crossed with y w; actin-Gal4/+ (heterozygous) flies and were allowed to lay eggs in different food as indicated. The flies from the F1 generation of this cross were counted, and an average from two different experiments is presented. $(D)$ Constitutive expression of Ctr1B rescues the developmental arrest and lethality of the $d M T F-1$ mutants under copper scarcity. $d M T F-1^{+/+}, y \mathrm{w}$ third instar larvae; $d M T F-1^{-/-}, d M T F-1^{140-1 R}$ mutant larvae (lethality under copper starvation occurs at second or third instar stages); $d M T F$ $1^{-/-}$and Ctr1B overexpression, $y$ w; $d M T F-1^{140-1 R}$, actin-Gal4/dMTF-1 ${ }^{140-1 R}$, UAS$C \operatorname{tr} 1 B$ larvae. Flies were allowed to deposit eggs in the respective food and larval pictures were taken $5 \mathrm{~d}$ after egg deposition. Bar, $1 \mathrm{~mm}$. 
starvation, the question remained whether the response was direct or indirect. Inspection of the upstream sequences of the $C \operatorname{tr} 1 B$ gene revealed a cluster of three metal response elements, designated MRE1-MRE3, and a fourth one set apart from them (Fig. 2B). To determine the significance of these MREs, we made a comparison to the several related species of Drosophila whose genome sequences are available in the database. We also amplified and sequenced the $C \operatorname{tr} 1 B$ genomic region from Drosophila virilis and included these data in our comparison. While the majority of upstream sequences have diverged considerably, the MRE cluster is highly conserved, both regarding the MREs themselves and their flanking sequences, among the four species (Supplementary Fig. 2). The comparisons also revealed that the fourth MRE, which lacks the typical flanking sequences of good MREs, is not conserved in the other Drosophila species. To test whether dMTF-1 can bind to the MREs of $\operatorname{Ctr1B}$, we conducted electrophoretic mobility shift assay (EMSA) experiments. Drosophila S2 cells were transfected with either Drosophila MTF-1 or human MTF-1 expression plasmids, and extracts from these cells were tested with radiolabeled oligonucleotides containing MREs from the Ctr1B upstream region. Indeed, both MRE1 and an oligo containing the closely spaced MRE2 and MRE3 of Ctr1B bound strongly to dMTF-1 and hMTF-1 and are well comparable to the binding of a consensus oligo designated MRE-s (Fig. 2A).

To narrow the region responsible for copper regula-
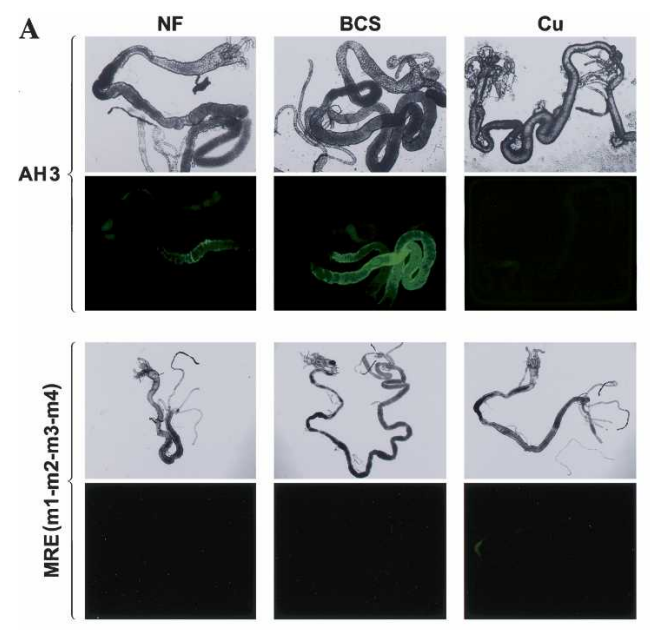

B

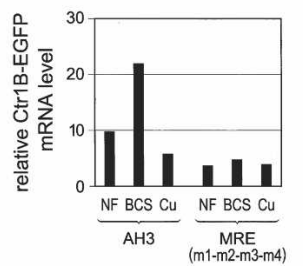

A

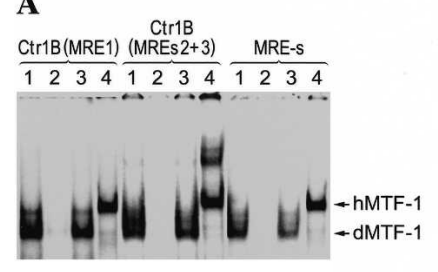

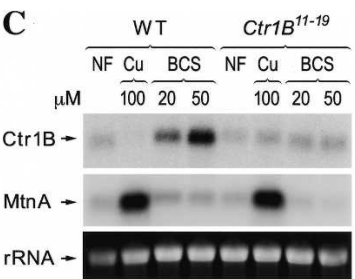

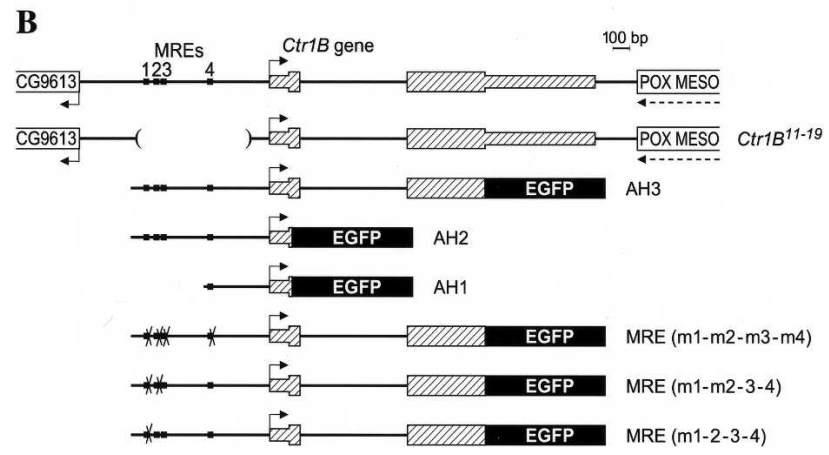

Figure 2. (A) MREs of Ctr1B bind MTF-1 very strongly. Drosophila S2 cells were transfected with dMTF-1 (lanes 1-3) or hMTF-1 (lane 4). (Lanes 2,3) Competitions with 200-fold excess of cold specific and nonspecific competitor oligos, respectively. Human MTF-1, due to its high proline content, migrates more slowly than dMTF-1. Note the double shift with the oligo containing MRE2 and MRE3. $(B)$ Schematic view of the $C \operatorname{tr} 1 B$ genomic region, $C \operatorname{tr} 1 B$ mutant $C \operatorname{tr} 1 B^{11-19}$ and constructs with either wild-type enhancer/promoter region (AH2, $\mathrm{AH} 3)$, large deletion (AH1), or specific mutations (MRE m1-m2-m3-m4; MRE m1-m2-3-4; MRE m1-2-3-4). (C) Ctr1B allele $\left(C \operatorname{tr} 1 B^{11-19}\right)$ with a deletion in the upstream region shows loss of copper regulation. RNA blotting analysis of total RNA obtained from third instar larvae at different conditions. (WT) wild type, y w; (NF) normal food; (rRNA) reference.
Figure 3. MREs and dMTF-1 are essential for up-regulation of Ctr1B upon copper depletion. (A) Transgenic flies with AH3 as a reporter show a clear up-regulation of green fluorescence in copper starvation. Induction is lost upon MRE mutations in the reporter constructs (lower panel) and also when AH3 transgenics were tested in the $d M T F-1$ mutant genetic background (BCS, $d M T F^{-1-}$. For details, see Supplementary Figure 3. (B) Quantification of EGFP transcripts by $\mathrm{S} 1$ nuclease protection assay from total RNA extracted from Drosophila third instar larvae. $(C)$ Plasma membrane localization of Ctr1B-EGFP fusion protein in the gut of transgenic larvae when fed with BCS. Nuclei were stained with Hoechst 33342 (blue).

tion, we tested transgenic flies with deletion constructs driving a fluorescent protein reporter. In one of these, the EGFP coding sequence was fused to the last codon preceding the stop codon of $C \operatorname{tr} 1 B$, thereby preserving not only the coding sequence but also the introns that might harbor regulatory sequences (AH3). In another construct, the first codon of $C \operatorname{tr} 1 B$ was fused to EGFP (AH2) (Fig. 2B). Transgene expression was found to be strongly induced in the larval gut by BCS-supplemented food (Fig. 3A, panel AH3; for AH2, see Supplementary Fig. 3A). Consistent with the role of Ctr1B in copper import, plasma-membrane-localized green fluorescence was observed in the cells of the larval gut of $\mathrm{AH} 3$ transgenic flies (Fig. 3C). Removal of the Ctr1B upstream region harboring the MRE1-MRE3 cluster (AH1) had a dramatic effect, in that the reporter gene was no longer inducible by copper depletion (Supplementary Fig. 3A). The quantification of the EGFP transcripts from whole larvae revealed a two- to threefold up-regulation of transcription in BCS-containing food (Fig. 3B; Supplementary Fig. 3B). In line with a role of MTF-1 in $C \operatorname{tr} 1 B$ regulation, there was no green fluorescence from $\mathrm{AH} 2$ and $\mathrm{AH} 3$ transgenes in the gut of $d M T F-1$ knockout larvae (Fig. 3A; Supplementary Fig. 3A). We also generated a genomic deletion of the Ctr1B locus by imprecise excision of an adjacent $\mathrm{P}$ element. One deletion of $685 \mathrm{bp}$ including the region that harbors the MREs was recovered (Fig. 2B). This $C \operatorname{tr} 1 B$ allele, designated $C \operatorname{tr} 1 B^{11-19}$, was no longer 
induced by copper starvation (Fig. 2C). For further elucidation of the role of MREs, we constructed several transgenic fly lines that contained point mutations in individual Ctr1B promoter MREs (Fig. 2B). The results with transgenic larvae showed that MREs are, indeed, critical for the up-regulation of $C \operatorname{tr} 1 B$ transcription under copper limiting conditions; these specific mutations abolished the expression in low copper, indistinguishable from a deletion of the entire cluster. Even the mutation of a single motif (MRE1) had the same detrimental effect (Fig. 3A; Supplementary Fig. 4).

To assess the biological importance of MREs in $C \operatorname{tr} 1 B$ gene regulation, we tested the ability of the Ctr1B constructs to rescue $C \operatorname{tr} 1 B$-null mutant flies in low and high copper concentrations. The results confirm the importance of the MREs in the $C \operatorname{tr} 1 B$ gene in that only the Ctr1B-EGFP construct with the wild-type promoter (AH3), but none of the constructs with MRE mutations, rescued the Ctr1B-null mutants from lethality in low copper (Table 1). These results lend further credence to a scenario in which $C \operatorname{tr} 1 B$ gene transcription is induced upon copper depletion via upstream MRE sequences and transcription factor MTF-1. As mentioned above, Ctr1Bnull mutants are also more sensitive to copper load than wild type. The exact reason for this remains to be elucidated; in any case, we find that the high-copper sensitivity can be rescued to a large extent even by a $C \operatorname{tr} 1 B$ transgene lacking the triple MREs. Thus, the main role of these MREs is in copper scarcity, rather than copper load.

Table 1. MREs are essential for the function of Ctr1B under copper starvation

\begin{tabular}{|c|c|c|c|c|c|c|c|c|c|}
\hline \multirow[b]{2}{*}{ Genotype } & \multirow[b]{2}{*}{ NF } & \multicolumn{8}{|c|}{ BCS concentration $(\mu \mathrm{M})$} \\
\hline & & 10 & 20 & 40 & 80 & 160 & 320 & 640 & 1280 \\
\hline$y w ;+/+;+/+$ & 66 & 67 & 68 & 65 & 66 & 57 & 46 & 36 & 23 \\
\hline$y w ;+/+; d M T F^{140-1 R}$ & 62 & 61 & 55 & 14 & 0 & 0 & 0 & 0 & 0 \\
\hline$y w ;+/+; C t r 1 B^{3-4}$ & 61 & 56 & 0 & 0 & 0 & 0 & 0 & 0 & 0 \\
\hline$y w ; p o x M R D ; C t r 1 B^{3-4}$ & 58 & 64 & 60 & 58 & 46 & 43 & 36 & 26 & 12 \\
\hline$y w ; A H 3 ; C \operatorname{tr} 1 B^{3-4}$ & 59 & 60 & 62 & 55 & 41 & 35 & 23 & 2 & 0 \\
\hline$y w ; M R E(m 1-m 2-m 3-m 4) ; C t r 1 B^{3-4}$ & 61 & 58 & 0 & 0 & 0 & 0 & 0 & 0 & 0 \\
\hline \multirow[t]{2}{*}{$y w ; M R E(m 1-m 2-3-4) ; C t r 1 B^{3-4}$} & 67 & 52 & 0 & 0 & 0 & 0 & 0 & 0 & 0 \\
\hline & & \multicolumn{8}{|c|}{ Cu concentration $(\mu \mathrm{M})$} \\
\hline Genotype & NF & 10 & 20 & 40 & 80 & 160 & 320 & 640 & 1280 \\
\hline $\boldsymbol{y} w ;+/+;+/+$ & 66 & 65 & 67 & 65 & 66 & 60 & 58 & 52 & 32 \\
\hline$y w ;+/+; d M T F^{140-1 R}$ & 62 & 65 & 60 & 58 & 42 & 16 & 0 & 0 & 0 \\
\hline$y w ;+/+; C \operatorname{tr} 1 B^{3-4}$ & 61 & 59 & 58 & 52 & 34 & 28 & 3 & 2 & 0 \\
\hline$y$ w; poxMRD; Ctr1B $B^{3-4}$ & 58 & 62 & 61 & 58 & 61 & 52 & 60 & 27 & 19 \\
\hline$y$ w; $A H 3 ; C t r 1 B^{3-4}$ & 59 & 64 & 63 & 60 & 52 & 48 & 52 & 24 & 9 \\
\hline$y w ; M R E(m 1-m 2-m 3-m 4) ; C t r 1 B^{3-4}$ & 61 & 63 & 53 & 55 & 59 & 43 & 32 & 14 & 0 \\
\hline$y w ; M R E(m 1-m 2-3-4) ; C \operatorname{Ct} 1 B^{3-4}$ & 67 & 61 & 56 & 61 & 38 & 26 & 12 & 6 & 0 \\
\hline
\end{tabular}

Genetic rescue shows the importance of MREs for Ctr1B activation under copper starvation. The reporter transgenes that carry the $C \operatorname{tr} 1 B$ gene fused to EGFP with the wild-type promoter and MRE mutations thereof were combined with the $C \operatorname{tr} 1 B$ null mutants. The poxMRD transgenic flies seerved as a positive control for the rescue experiment; the poxMRD construct carries the complete $C \operatorname{tr} 1 B$ genomic region including upstream and downstream genes without EGFP fusion. The flies were allowed to lay 200 eggs in the respective food and the parents were removed after $4 \mathrm{~d}$. Results are the mean of three independent experiments and the numbers indicate the percentage of adult flies eclosing.
The results obtained so far demonstrate that dMTF-1 is not only essential for the activation of metallothioneins and other target genes upon heavy metal load, but also to regulate transcription of $C \operatorname{tr} 1 B$ under copper starvation (see also Supplementary Fig. 5). Because in the case of other transcription factors, subtle differences in the DNA-binding site can determine whether the factor interacts with a coactivator or a corepressor (e.g., Dostert and Heinzel 2004), we considered it possible that the MRE motifs of $C \operatorname{tr} 1 B$ themselves could bring about transcriptional induction at low copper. To this end, we generated two types of reporter transgenes with a synthetic "minipromoter," one containing the four MRE motifs from the $C \operatorname{tr} 1 B$ gene with hardly any intervening sequences, arranged in tandem arrays, and another one where only MRE1 was multimerized to four copies (Fig. 4A). We compared these two reporter transgenes with a similar synthetic minipromoter, which contains a tandem array of MRE motifs derived from the metallothionein B $(M \operatorname{tn} B)$ gene (Zhang et al. 2001). Interestingly, all three reporter transgenes behaved like a genuine metallothionein promoter: They were strongly induced when the larvae were fed with copper, but were not responsive to low copper (Fig. 4B; data not shown). Also in cell culture, all three reporter constructs were robustly induced by copper treatment (Fig. 4C). Thus, the $C \operatorname{tr} 1 B$ MREs on their own are not sufficient to confer transcriptional induction upon copper depletion, but rather respond to metal load. This suggests that sequences in addition to MREs in the $C \operatorname{tr} 1 B$ enhancer/promoter region contribute to the regulatory characteristics of that gene.

How could one transcription factor exert two diametrically different functions? One possibility could be that this special architecture of the MREs in the $C \operatorname{tr} 1 B$ enhancer/promoter facilitates cooperative binding between dMTF-1 and a hypothetical copper-dependent repressor protein. Under normal conditions, this repressor would be partially removed, resulting in a moderate expression, while under copper starvation it would dissociate from dMTF-1 completely, yielding higher expression. Further experiments will be required to elucidate the exact mechanism of $C \operatorname{tr} 1 B$ activation via dMTF-1 under conditions of copper starvation.

How do other organisms handle copper excess and copper starvation? In the yeast Saccharomyces cerevisiae, the two extremes require different transcription factors. The homologs of Ctrl that import copper are activated upon copper starvation by the Mac1 transcription factor (Yamaguchi-Iwai et al. 1997); the activation of metallothionein genes upon copper load is driven by the transcription factor Acel (Thiele 1988; Winge 1998; Rutherford and Bird 2004). In mammals, there are two Ctr homologs, Ctr1 and Ctr2. Neither of them is apparently regulated at the level of transcription by copper availability (Lee et al. 2001, 2002), and we also did not find any MREs in their enhancer/promoter region (data not shown). In conclusion, the major role of MTF-1 is to handle heavy metal load; accordingly, MREs are found in the metallothionein genes and other metal-responsive genes from insects to mammals. In contrast, regulation of the $C \operatorname{tr} 1 B$ copper importer via MREs/MTF-1 appears to have evolved specifically in Drosophilidae as an efficient way to cope with copper starvation. This represents a novel regulatory mechanism in which one and the same transcription factor serves as an activator of different genes in response to opposite environmental conditions. 
A
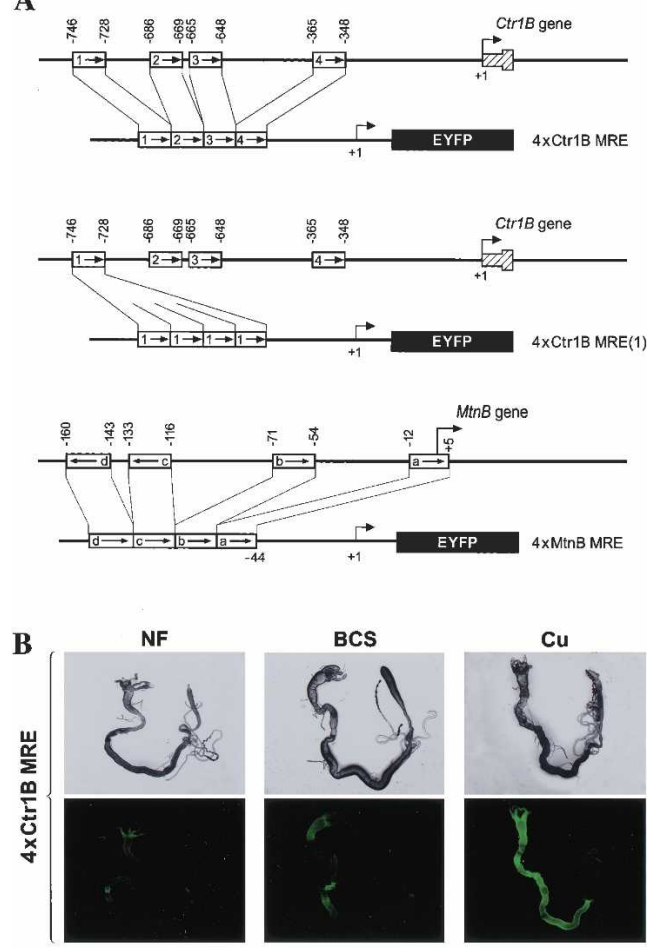

C

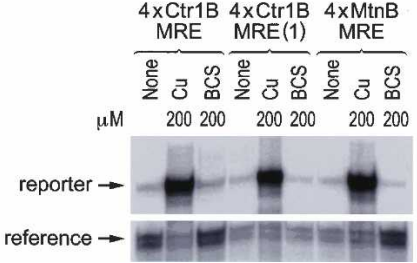

Figure 4. MRE motifs from metallothionein $M t n B$ and also from copper importer $C \operatorname{tr} 1 B$ confer high copper induction. $(A)$ Minipromoters containing four tandem MREs of the $C \operatorname{tr} 1 B$ upstream region (upper construct), four copies of Ctr1B MRE1 (middle construct), or four MREs from the $M \operatorname{tn} B$ upstream region (lower construct) fused to the minimal heterologous promoter from the $h s p 70$ gene. $(B)$ Minipromoter containing four MRE motifs from the Ctr1B gene up-regulates the reporter transgene in high copper, similar to the metallothionein promoter. $(C)$ Minipromoters containing four MREs of the $C \operatorname{tr} 1 B$ upstream region, four copies of $C \operatorname{tr} 1 B$ MRE1, or four MREs from the $M t n B$ upstream region were assembled into an OVEC reporter construct. The constructs were transfected into Drosophila S2 cells, and total RNA was subjected to S1 nuclease protection assay. (Reference) Endogenous tubulin $\alpha 1$ transcripts.

\section{Materials and methods}

Fly food, fly stocks, and genetics

Flies were raised on standard cornmeal-based food; $200 \mu \mathrm{M}$ BCS or 200 $\mu \mathrm{M}$ copper was supplemented to the food for all the fluorescence analyses except for the experiment with the $\mathrm{AH} 3$ and $\mathrm{AH} 2$ transgene in a dMTF-1 mutant background, where $40 \mu \mathrm{M}$ BCS was used. Both AH3 and AH2 are well inducible at $40 \mu \mathrm{M}$ BCS (data not shown). The homozygous null allele for $d M T F-1\left(d M T F-1^{140-1 R}\right)$ is indicated throughout the manuscript as $d M_{T F-1^{-1-}}$. The $\mathrm{EP}(3) 0833$ line, which harbors a single $\mathrm{P}$ element 800 bp upstream of the $C \operatorname{tr} 1 B$ transcription start site, was obtained from the Bloomington Stock Center. To generate a Drosophila Ctr1B promoter deletion (allele $C \operatorname{tr} 1 B^{11-19}$ ), an imprecise P-element excision strategy was used as described in Zhou et al. (2003). A complete list of fly stocks generated is included in the Supplemental Material.
DNA constructs

See Supplemental Material.

GFP expression analysis and microscopy

For the EGFP or EYFP reporter analysis, flies were allowed to deposit eggs in the food and raised until third instar larvae. The larval gut was dissected and analyzed under a Leica DRB fluorescence stereomicroscope. The images in Figure 3A are magnified 5x, and all the other larval gut images were made at $2.5 \times$ magnification. For the subcellular localization of Ctr1B, Drosophila larval gut was analyzed at $63 \times$ magnification using a LEICA TCS SP spectral confocal microscope.

Cell culture and transient transfection assay

Drosophila S2 cells were grown at $25^{\circ} \mathrm{C}$ under standard culture conditions. Various OVEC reporter constructs driven by Drosophila promoters were transfected together with an expression vector for dMTF-1 driven by the Drosophila actin5c promoter using the calcium-phosphate coprecipitation method (Westin et al. 1987). Seventy-two hours post-transfection, cells were treated with the indicated concentrations of $\mathrm{CuSO}_{4}$ or $\mathrm{BCS}$ and incubated for another $24 \mathrm{~h}$ before harvesting.

RNA extraction, S1 nuclease protection assay, and RNA blotting Flies with various genotypes were grown in normal, BCS-supplemented, or copper-supplemented food, and the third instar larvae were harvested for total RNA extraction using the TRIzol reagent (Invitrogen). The S1 nuclease protection assay was performed using $100 \mu \mathrm{g}$ of total RNA as described previously (Weaver and Weissmann 1979). The gels were developed using a PhosphorImager, and bands were quantified using ImageQuaNT software. For quantification of EGFP transcripts, endogenous actin5c was used for normalization. RNA blotting experiments were performed using the $C \operatorname{tr} 1 B$ and $M \operatorname{tnA}$ cDNA as ${ }^{32} \mathrm{P}$-labeled probes. rRNA (reference) was stained with ethidium bromide.

Electrophoretic mobility shift assay (EMSA)

Transient transfections in Drosophila S2 cells were carried out as mentioned above. The nuclear extracts were prepared and EMSA was performed as described previously (Radtke et al. 1993). Binding reactions were performed by incubating $25 \mathrm{fmol}$ of ${ }^{32} \mathrm{P}$ end-labeled, a 28-bp-long oligonucleotide containing the MRE1 motif from Ctr1B promoter, a 40bp-long oligonucleotide containing the closely spaced MRE2 and MRE3 from Ctr1B, or a 31-bp-long DNA oligonucleotide containing an MRE core consensus sequence TGCACAC designated MRE-s (Radtke et al. 1993) as a positive control for MTF-1 binding. For competition experiments, 5 pmol of unlabeled oligo (either specific or nonspecific) was added to the reaction mixture prior to addition of the extracts. For complete oligonucleotide sequences, see Supplemental Material.

Database searches and computer analysis of the sequences Database homology searches were carried out using the University of California, Santa Cruz, blat server (http://www.genome.ucsc.edu). The alignment was done in CLUSTALW.

\section{Acknowledgments}

We are greatly indebted to Fritz Ochsenbein for the preparation of figures. We thank Antonia Manova and Bruno Schmid for technical assistance. We also thank Jianming Chen and Markus Noll for the poxMRD flies, Hajime Takeuchi and Janis Bennion for critical reading of the manuscript, and Ken Cadigan for helpful advice. This work was supported by the Swiss National Science Foundation, the Kanton Zürich, and a grant from the United States National Institutes of Health (GM62555) to D.J.T.

\section{References}

Askwith, C.C. and Kaplan, J. 1998. Site-directed mutagenesis of the yeast multicopper oxidase Fet3p. J. Biol. Chem. 273: 22415-22419.

Balamurugan, K., Egli, D., Selvaraj, A., Zhang, B., Georgiev, O., and Schaffner, W. 2004. Metal-responsive transcription factor (MTF-1) and heavy metal stress response in Drosophila and mammalian cells: A functional comparison. Biol. Chem. 385: 597-603.

Bertinato, J. and L'Abbe, M.R. 2004. Maintaining copper homeostasis: Regulation of copper-trafficking proteins in response to copper deficiency or overload. J. Nutr. Biochem. 15:316-322. 
Culotta, V.C., Lin, S.J., Schmidt, P., Klomp, L.W., Casareno, R.L., and Gitlin, J. 1999. Intracellular pathways of copper trafficking in yeast and humans. Adv. Exp. Med. Biol. 448: 247-254.

Dostert, A. and Heinzel, T. 2004. Negative glucocorticoid receptor response elements and their role in glucocorticoid action. Curr. Pharm. Des. 10: 2807-2816.

Egli, D., Selvaraj, A., Yepiskoposyan, H., Zhang, B., Hafen, E., Georgiev, O., and Schaffner, W. 2003. Knockout of 'metal-responsive transcription factor' MTF-1 in Drosophila by homologous recombination reveals its central role in heavy metal homeostasis. EMBO J. 22: 100108.

Giedroc, D.P., Chen, X., and Apuy, J.L. 2001. Metal response element (MRE)-binding transcription factor-1 (MTF-1): Structure, function, and regulation. Antioxid. Redox Signal. 3: 577-596.

Günes, C., Heuchel, R., Georgiev, O., Muller, K.H., Lichtlen, P., Bluthmann, H., Marino, S., Aguzzi, A., and Schaffner, W. 1998. Embryonic lethality and liver degeneration in mice lacking the metal-responsive transcriptional activator MTF-1. EMBO J. 17: 2846-2854.

Halliwell, B. and Gutteridge, J.M. 1985. The importance of free radicals and catalytic metal ions in human diseases. Mol. Aspects Med. 8: 89-193.

Harrison, M.D. and Dameron, C.T. 1999. Molecular mechanisms of copper metabolism and the role of the Menkes disease protein. J. Biochem. Mol. Toxicol. 13: 93-106.

Heuchel, R., Radtke, F., Georgiev, O., Stark, G., Aguet, M., and Schaffner, W. 1994. The transcription factor MTF-1 is essential for basal and heavy metal-induced metallothionein gene expression. $E M B O J$. 13: $2870-2875$

Kägi, J.H. and Kojima, Y. 1987. Chemistry and biochemistry of metallothionein. Experientia Suppl. 52: 25-61.

Lane, C., Petris, M.J., Benmerah, A., Greenough, M., and Camakaris, J. 2004. Studies on endocytic mechanisms of the Menkes copper-translocating P-type ATPase (ATP7A; MNK). Endocytosis of the Menkes protein. Biometals 17: 87-98.

Langmade, S.J., Ravindra, R., Daniels, P.J., and Andrews, G.K. 2000. The transcription factor MTF-1 mediates metal regulation of the mouse ZnT1 gene. J. Biol Chem. 275: 34803-34809.

Lee, J., Prohaska, J.R., and Thiele, D.J. 2001. Essential role for mammalian copper transporter Ctrl in copper homeostasis and embryonic development. Proc. Natl. Acad. Sci. 98: 6842-6847.

Lee, J., Petris, M.J., and Thiele, D.J. 2002. Characterization of mouse embryonic cells deficient in the ctrl high affinity copper transporter. Identification of a Ctrl-independent copper transport system. J. Biol. Chem. 277: 40253-40259.

Lichtlen, P. and Schaffner, W. 2001. Putting its fingers on stressful situations: The heavy metal-regulatory transcription factor MTF-1. Bioessays 23: 1010-1017.

Mercer, J.F. 2001. The molecular basis of copper-transport diseases. Trends Mol. Med. 7: 64-69.

Palmiter, R.D. 1998. The elusive function of metallothioneins. Proc. Nat1. Acad. Sci. 95: 8428-8430.

Petris, M.J., Smith, K., Lee, J., and Thiele, D.J. 2003. Copper-stimulated endocytosis and degradation of the human copper transporter, hCtr1. J. Biol. Chem. 278: 9639-9646.

Puig, S. and Thiele, D.J. 2002. Molecular mechanisms of copper uptake and distribution. Curr. Opin. Chem. Biol. 6: 171-180.

Radtke, F., Heuchel, R., Georgiev, O., Hergersberg, M., Gariglio, M., Dembic, Z., and Schaffner, W. 1993. Cloned transcription factor MTF-1 activates the mouse metallothionein I promoter. EMBO J. 12: 1355-1362.

Rutherford, J.C. and Bird, A.J. 2004. Metal-responsive transcription factors that regulate iron, zinc, and copper homeostasis in eukaryotic cells Eukaryot. Cell 3: 1-13.

Stuart, G.W., Searle, P.F., and Palmiter, R.D. 1985. Identification of multiple metal regulatory elements in mouse metallothionein-I promoter by assaying synthetic sequences. Nature 317: 828-831.

Thiele, D.J. 1988. ACE1 regulates expression of the Saccharomyces cerevisiae metallothionein gene. Mol. Cell. Biol. 8: 2745-2752.

Waggoner, D.J., Bartnikas, T.B., and Gitlin, J.D. 1999. The role of copper in neurodegenerative disease. Neurobiol. Dis. 6: 221-230.

Wang, Y., Wimmer, U., Lichtlen, P., Inderbitzin, D., Stieger, B., Meier, P.J., Hunziker, L., Stallmach, T., Forrer, R., Rulicke, T., et al. 2004. Metal-responsive transcription factor-1 (MTF-1) is essential for em- bryonic liver development and heavy metal detoxification in the adult liver. FASEB J. 18: 1071-1079.

Weaver, R.F. and Weissmann, C. 1979. Mapping of RNA by a modification of the Berk-Sharp procedure: The $5^{\prime}$ termini of $15 \mathrm{~S} \beta$-globin mRNA precursor and mature $10 \mathrm{~S} \beta$-globin mRNA have identical map coordinates. Nucleic Acids Res. 7: 1175-1193.

Westin, G. and Schaffner, W. 1988. A zinc-responsive factor interacts with a metal-regulated enhancer element (MRE) of the mouse metallothionein-I gene. EMBO I. 7: 3763-3770.

Westin, G., Gerster, T., Muller, M.M., Schaffner, G., and Schaffner, W. 1987. OVEC, a versatile system to study transcription in mammalian cells and cell-free extracts. Nucleic Acids Res. 15: 6787-6798.

Winge, D.R. 1998. Copper-regulatory domain involved in gene expression. Prog. Nucleic Acid Res. Mol. Biol. 58: 165-195.

Yamaguchi-Iwai, Y., Serpe, M., Haile, D., Yang, W., Kosman, D.J., Klausner, R.D., and Dancis, A. 1997. Homeostatic regulation of copper uptake in yeast via direct binding of MACl protein to upstream regulatory sequences of FRE1 and CTR1. J. Biol. Chem. 272: 17711-17718.

Zhang, B., Egli, D., Georgiev, O., and Schaffner, W. 2001. The Drosophila homolog of mammalian zinc finger factor MTF-1 activates transcription in response to heavy metals. Mol. Cell. Biol. 21: 4505-4514.

Zhou, H., Cadigan, K.M., and Thiele, D.J. 2003. A copper-regulated transporter required for copper acquisition, pigmentation, and specific stages of development in Drosophila melanogaster. J. Biol. Chem. 278: $48210-48218$. 


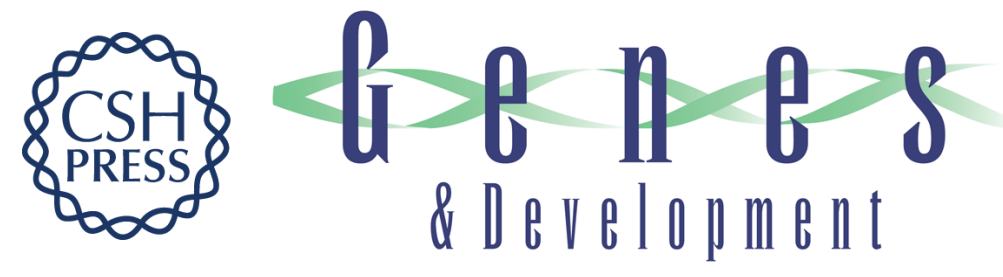

\section{Metal-responsive transcription factor (MTF-1) handles both extremes, copper load and copper starvation, by activating different genes}

Anand Selvaraj, Kuppusamy Balamurugan, Hasmik Yepiskoposyan, et al.

Genes Dev. 2005, 19:

Access the most recent version at doi:10.1101/gad.1301805

Supplemental http://genesdev.cshlp.org/content/suppl/2005/04/01/19.8.891.DC1
Material

References This article cites 33 articles, 12 of which can be accessed free at: http://genesdev.cshlp.org/content/19/8/891.full.html\#ref-list-1

License

Email Alerting

Receive free email alerts when new articles cite this article - sign up in the box at the top Service right corner of the article or click here.

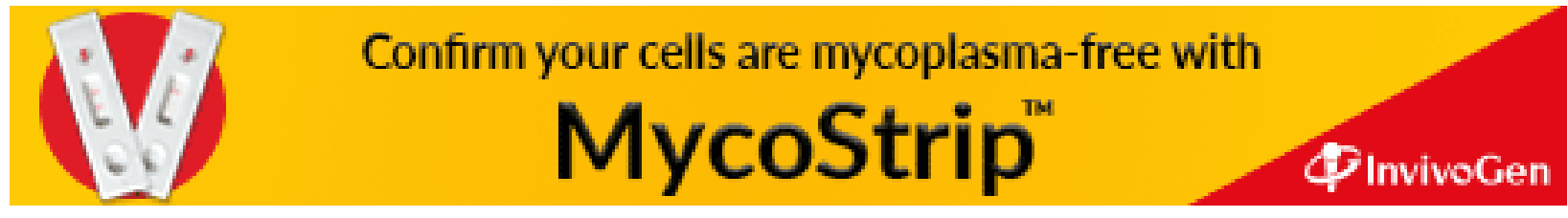

